\title{
An Adaptive Reputation-based Trust Framework for Peer-to-Peer Applications
}

\author{
William Sears, Zhen Yu, and Yong Guan \\ Department of Electrical and Computer Engineering \\ Iowa State University, Ames, Iowa 50011 \\ Email: sears@ ameslab.gov and \{yuzhen, yguan\}@ iastate.edu
}

\begin{abstract}
In distributed $P 2 P$ environments, peers (i.e., users) often have to request the services from some unfamiliar peers (i.e., resources) that could be altruistic, selfish, or even malicious. To motivate selfish peers to cooperate and minimize the risk from malicious peers, we propose an adaptive reputation-based trust framework for peer-to-peer applications. In this framework, we define a quantifiable metric trust that is calculated from the user's own view as well as other peers' view (as references) on the reputation of a peer (resource). It can be used to quantify the trustworthiness of peers and provide a measurable and trustable way of resource scheduling/selection and access control for peer-topeer applications. We assume economic resources compete with each other to maximize their profit and design a number of strategies for resources to determine their price. The simulation results show that the framework supports economic resources to achieve long-term high user satisfaction, differentiates malicious nodes from normal ones and encourages the resources to provide high-quality services.
\end{abstract}

\section{Introduction}

In distributed $\mathrm{P} 2 \mathrm{P}$ applications, we can differentiate the roles of peers as user nodes and resource nodes (or users and resources for short). Resource nodes provide services utilizing their resources such as shared files, memory, or processors to users, while the users submit their jobs, e.g., to download some files, to request for computation or usage of some types of resources. We study the resource scheduling/selection for P2P applications, which can be further divided into two problems: (1) How to guarantee that users schedule their jobs to the most reliable resource nodes, especially when there exist some selfish and malicious resource nodes in the systems; (2) How to motivate the resource nodes to provide high-quality and reliable resources to the users.

The first problem has attracted a lot of research, most of which are based on reputation and trust for resources scheduling. Selcuk, et al. defined several types of malicious resource nodes in [18] and proposed a reputationbased trust management scheme for P2P networks. Song and Hwang [19] first applied Fuzzy Logic for computing trust values in order to achieve security assurance and resource optimization in Grid computing systems. Azzedin, et al. [4] suggested that the trust values decay with time and trust relationships be based on a weighted combination of the direct relationships between domains as well as on the global reputation of the domain. Kamvar, et al. proposed the EigenTrust algorithm [10] for reputation management in P2P networks. One problem in these papers is that they did not clearly differentiate reputation from trust. Reputation and trust are generally confused either as a probability with which the resource is expected to complete a job, or as a mechanism to help users to obtain such a probability. Some papers even referred to trust rating as the evaluation result of the transactions between users and resources. Such definition for trust is not accurate for many real-world scenarios.

For the second problem, there are less research done. A few questions are raised, for example, why the resource nodes should provide their resources to the users selflessly, and why they should risk themselves being attacked for providing their resources without any benefit. A similar problem in file-sharing P2P applications such as Gnutella [8] and Kazaa [11] is free-riding due to the existence of free-riders who only want to download files from others but without sharing their files. Golle, et all. proposed a micro-payment mechanism [7] to encourage file sharing in P2P systems. Ramaswamy and Liu also discussed using utility functions [14] to measure the usefulness of the users. Although our purpose is not to solve free-riding and achieve fairness in P2P systems, we use the same idea to solve the second problem, i.e., users should give rewards or incentives to the resources that provide satisfactory services.

In this paper, we propose an adaptive reputation-based trust framework for peer-to-peer applications. In distributed environments such as P2P networks or Grid computing systems, peers (i.e., users) often have to request the services from some unfamiliar peers (i.e., resources) that could be altruistic, selfish, or even malicious. Therefore, it is desirable to design an adaptive trust model to motivate cooper- 
ativeness of selfish peers and minimize the risk from malicious peers. We assume the resource nodes are economic or selfish and the users should make payments for using the resources. Economic and selfish nodes aim at maximizing their profit. The non-malicious resource nodes may compete with each other to gain as much profit as possible. Different from traditional reputation-based trust systems, we categorize the roles of peers into three: users, references, and resources. A peer can take one or more of these three roles at the same time within its application context. We define three basic quantifiable metrics: job satisfactory ratings, reputation, and trust, where reputation is calculated from the user's historical transaction-based job satisfactory ratings and trust is calculated from the user's own view as well as other peers' view (as references) on the reputation of a peer (resource). The metric trust can be used to quantify the trustworthiness of peers and provide a trustable way of resource scheduling/selection and access control for peerto-peer applications. The simulation results show that our framework supports economic resources to achieve longterm high user satisfaction, differentiates malicious nodes from normal ones and encourages the resources to provide high-quality services.

\section{Related Work}

Selcuk, et al. [18] designed a protocol for P2P systems in which a peer looking to download a file uses reputation from itself and others to help determine which host to download from. Meanwhile, the authors defined four types of malicious resources such as Naive, Hypocritical, Collaborative, and PseudoSpoofing. The authors compared the groups of peers that offer different versions of the same file first and then selects one node from a group to download the file from. The protocol is too extreme in the evaluation as it appears there is only one criteria for rating a peer depending on whether the file is correct or not. However, other criteria could be examined such as duration of download. In addition, when a peer is selecting a group to download a file from, it is possible that there may exist an overwhelming majority of positively rated peers in one group only to be tainted by a single peer with a positive distrust rating.

Ramaswamy and Liu [14] discussed the free riding problem that infests many peer-to-peer systems. They proposed an incentive system that is aimed at keeping peers on the network to share their files based on three characteristics: the amount of files shared, the amount of data the peer has shared, and the popularity of the shared file. Peers are thus rewarded based on these metrics. The authors however did not address the spoofing that may occur when a peer wishes to gain access to the system. It would be possible for the peer to create many files with the name of a popular share in order to receive the reward which is additional download capability. Implementing file hashes would solve one problem, but does not prevent the peer from putting up a sig- nificant amount of large sized files in order to enhance its ability to download from the system.

Kamvar, et al. [10] described an algorithm for maintaining and receiving reputation values from peers in both a distributed and non-distributed manner. The algorithm collects reputation values from other peers and their peers, etc. and then normalizes the end result in which the user is then able to make a decision regarding which resource to use. This system also relies on a set of pre-defined trusted peers in order for the system to be most effective. The problem with using pre-defined trusted peers is that if one were to turn malicious or get hacked, it would have a negative effect on the entire system similar to that of a house with a crumbling foundation. However, if the pre-defined peers remain untainted, the system is very effective at eliminating the use of malicious peers. Another problem of this algorithm is the amount of iterations required to receive trust values becomes increasingly lengthy as the size of the entire system increases, which reduces the scalability of the algorithm.

\section{Problem Statement}

In this section, we discuss the system model and threat model. Then, we present the four primary goals of our adaptive reputation-based trust framework for $\mathrm{P} 2 \mathrm{P}$ applications.

\subsection{System Model}

In P2P applications, the roles of peers can be categorized as resource nodes and user nodes. Resource nodes provide services utilizing their resources such as shared files, memory and processors for users. We model the systems as a graph $G=(V, E)$, where $V$ and $E$ are the set of vertices and edges. Each vertex represents a node in the systems. Moreover, we have $V=V_{u} \cup V_{r s}$, where $V_{u}$ is the set of users and $V_{r s}$ is the set of resource nodes. We also define $E=\left\{\langle x, y\rangle \mid x \in V_{u}, y \in V_{r s}\right\}$, where the edge $\langle x, y\rangle$ denotes the interaction or relationship between user $x$ and resource $y$. Compared to the traditional distributed systems, P2P systems has a distinct property that the roles of resources and users are not static. Physically, a particular node can be either a user or a resource, or even both at the same time, i.e., the node can provide its resource to others and simultaneously submit its own job to request the resources from others.

To motivate resources to provide high-quality services to users, we assume users pay for the jobs they submitted. Let us consider such a scenario: Before each user submits its jobs, it queries the system for available resources that can fulfil its jobs. Receiving the query, those resources capable and willing to process the jobs would respond to the user with the desired payment (or price) for processing the jobs. Ideally, users and resources are economic. Thus, an economic user would select one or multiple best resources to process its jobs (We will discuss how to select the best ones later.). Meanwhile, the economic resources would compete 
with each other by offering an appropriate price to win the user's selection in order to maximize their profit.

\subsection{Threat Model}

We assume users pay before using resources, hence, there is no way for users to repudiate. However, there do exist some selfish or malicious resources that refuse to provide satisfactory services or even attack the users by providing malicious resources, e.g., offering a shared file that contains malicious code after receiving the users' payments. Therefore, we must identify these possible threats.

- Honest Resource $\left(V_{r s_{-} H}\right)$ : An honest resource is not malicious. However, it attempts to undercut the competition by always offering the lowest possible price and performing jobs to a satisfactory level. Its goal is to be a monopoly to the distributed systems equivalent to Wal-Mart in the retail trade. This would be very dangerous because it can cause severe damages to the system if it becomes malicious.

- Naive Resource $\left(V_{r s_{-} N}\right)$ : A naive resource always offers the lowest price to gain the selection of users, however, it never provides the services required by the users. Its goal is to disturb the system as much as possible by always providing bad services to users.

- Random and Structured Hypocritical Resource $\left(V_{r s_{-} H y R}\right.$ and $\left.V_{r s_{-} H y S}\right)$ : Hypocritical resources provide satisfactory service a majority of the time, however, they are deemed malicious because they purposely provide bad service at a set rate. There are two types of hypocritical resources, random and structured. The random hypocritical resource acts maliciously with some probability, e.g., $20 \%$. On the contrary, a structured hypocritical resource does not become malicious based on chance, but follows some pattern, e.g., provide one malicious service after every four good ones.

- Collaborative Resource $\left(V_{r s_{-} C}\right)$ : Resource nodes and user nodes may be collaborative and form a special group. They act differently depending on whether the requesting peers are in the same group. For example, a collaborative resource may provide satisfactory services to a user (or insider) from the same group, but refuse to serve or even provide malicious services to an outsider. We can categorize collaborative resources into different types based on three aspects such as Resource Availability, Resource Price and Resource Performance. These aspects specify whether the resource is available to the outsider, what price (low, normal, or high) and what quality of service (normal, malicious) it offers to the outsider.

Let $V_{r s_{-} E}$ denote the set of economic (or normal) resources. We divide the resource nodes into various types such that $V_{r s}=V_{r s_{-} E} \cup V_{r s_{-} H} \cup V_{r s_{-} N} \cup V_{r s_{-} H y R} \cup V_{r s_{-} H y S} \cup V_{r s_{-} C}$.

\subsection{Goals}

Considering the P2P systems of users and various types of resources, we study the resource management and scheduling problem, i.e., how to efficiently motivate resources to provide satisfactory services to users and how to guide users to select the most reliable resources to fulfill their jobs, especially when there exist malicious resources. We expect to design a framework for distributed P2P applications and achieve the following goals: (1) Motivate the economic and non-malicious resources to provide the best services as possible; (2) Reduce the impact that malicious resources have on the system as a whole; (3) Provide a facility for an economic user access the best resources possible; (4) Allow per usage contracts for greater flexibility compared to long term contracts.

\section{A Framework Supporting Reputation- based Trust for P2P Applications}

\subsection{Overview}

In our framework, we introduce three roles of peers: user, resource and reference. A reference is a node from whom a user can ask for opinion about a resource. The references are needed because the user may not have any experience with that resource it wants to evaluate. After each job is done, a user calculates a job rating to evaluate the quality of the job finished. Based on a sequence of historical job ratings, the user can calculate the reputation of each resource to measure the overall quality of services provided by the resource. Moreover, combining the job ratings provided by the references, the user can calculate the trust value of each resource, which indicates the probability that the user can expect the resource to provide a satisfactory service for its next job. Considering both the price and trust of resources, the user can calculate and compare the appraisal to select the most appropriate (or reliable) resource to fulfill its job. Meanwhile, all economic resources compete with each other to win the user's selection. We design a number of strategies for resources to determine the price they should offer in order to maximize their profit in these long term competitions.

\subsection{Job Rating}

A job contains the requirements for some resources. It may involve the types of resources needed, the deadline to finish the job, whether it needs encryption, or any other requirements. A transaction between a user and a resource is referred as the processing of a job. After receiving the result of a job, the user calculates a job rating to evaluate the quality of this job finished. In our framework, the job rating is a value between zero and one, where $b_{r s}(x, y)$ denotes the job rating of user $x$ for resource $y$. (In some papers, job rating is named as trust rating and may be a binary value.)

\subsection{Reputation}

The reputation of a user on a resource is a metric measuring the overall quality of previous transactions between 
the resource and the user. In our framework, it is a value between zero and one, calculated from the job ratings recorded by the user. (In other papers, it is called local trust, which we believe confuses the trust and the reputation and is not accurate.) Assume user $x$ stores up to $n$ job ratings of previous transactions with resource $y$, the reputation $r_{r s}(x, y)$ is:

$$
r_{r s}(x, y)=\frac{\sum_{i=1}^{n} \alpha^{i} \cdot b_{r s, i}(x, y)}{\sum_{i=1}^{n} \alpha^{i}},
$$

where $b_{r s, i}(x, y)$ denotes the $i$-th job rating and the first rating corresponds to the most recent job. $\alpha \in(0,1]$ is a decay factor, which indicates how important the most recent transaction is to the reputation. Note: Each user may set a different value of $\alpha$ for itself.

\subsection{Reference}

Before submitting its jobs, a user needs to evaluate the resources to select the most appropriate one for processing the jobs. Since the user may not have any transaction or sufficient number of transactions with the resource, the user could ask the opinions of other nodes, called references, to gain an overall evaluation of the resource. Let $V_{r f}$ denote the set of references. We have three types of nodes in our system model such that $V=V_{u} \cup V_{r s} \cup V_{r f}$. Physically, each node can be a user, resource, or reference, except that any resource cannot be the reference to the same type of resources. As for the resources, we assume the users pay the references to buy the information from these references, and the references compete with each other to sell their information to the users.

\subsection{Trust}

In our framework, the information provided by a reference is just the job ratings recorded by the reference for some resource. Hence, the user can use these job ratings to calculate a trust value of the resource. We define trust as the probability with which the user expects the resource to finish its next job. Assuming user $x$ receives the job ratings for a particular resource $y$ from $n$ reference $z_{i}(i=1, \cdots, n)$ and all nodes use the same decay factor $\alpha$, thus user $x$ can compute the reputation $r_{r s}\left(z_{i}, y\right)$ of each reference $z_{i}$ to resource $y$ and the corresponding trust $\operatorname{tr}(x, y)$ of this user $x$ to resource $y$ :

$$
\operatorname{tr}(x, y)=\frac{r(x, y)+\sum_{i=1}^{n} r_{r f}\left(x, z_{i}\right) \cdot r\left(z_{i}, y\right)}{1+\sum_{i=1}^{n} r_{r f}\left(x, z_{i}\right)}
$$

where $r_{r f}\left(x, z_{i}\right)$ denotes the reputation of user $x$ on reference $z_{i}$. In (2), we weight the reputation $r_{r s}\left(z_{i}, y\right)$ by $r_{r f}\left(x, z_{i}\right)$. This illustrates how reliable the user believes the information from each reference $z_{i}$.

\subsection{Appraisal}

After computing the trust of each resource and receiving the price offered by each resource, the user should select the most appropriate resource to fulfill its job. Generally, the user would like to choose a reliable resource offering an acceptable price. In our framework, we define appraisal as a metric for the user to select the most appropriate resource. Considering both the price and the quality of service, we define the appraisal $v(x, y)$ of user $x$ for resource $y$ as:

$$
v(x, y)=\operatorname{pr}(y) \cdot(1-\operatorname{tr}(x, y)),
$$

where $\operatorname{pr}(y)$ denotes the price offered by resource $y$. The resource having the lowest value of appraisal would be selected. Hence, appraisal brings a balance between the quality of service and the price offered by resources.

\subsection{Adaptive Adjustment of Reference's Reputa- tion}

We should notice that the references can also be malicious. They may provide false information to mislead the user to choose a poor or malicious resource. Similar to the classification of resources, we can define various types of references such as Economic, Naive, Hypocritical and Collaborative ones.

Therefore, the users also need a way to evaluate the references in order to mitigate against the malicious ones. Since the information provided by the reference helps the user predict the quality of service for the resource, the closer the information is to the real result, the more reliable the reference would be. For some transaction between user $x$ and resource $y$, we define the rating $b_{r f}(x, z)$ of user $x$ to reference $z$ as follows:

$$
b_{r f}(x, z)=1-\left|b_{r s}(x, y)-r_{r s}(z, y)\right|,
$$

where $b_{r s}(x, y)$ denotes the job rating of this transaction between $x$ and $y$, and $r_{r s}(z, y)$ is the reputation of reference $z$ on resource $y$, which is computed by user $x$ from the information collected from $z$. (If $x$ and $z$ have different decay factor $\alpha, x$ should compute $\bar{r}(z, y))$ based on its own $\alpha$.) $b_{r f}(x, z)$ will be stored by user $x$ and used to update $r_{r f}(x, z)$ by the equation similar to (1).

\subsection{Strategies for Resources to Achieve Their Profit Goal}

A resource receives the payment from a user for processing its job. Except for malicious resources, a normal one must be economic, i.e., the purpose of an economic resource is to maximize its profit. In the following sections, we will discuss a number of strategies for resources to fulfill this purpose.

\subsubsection{Binary and Exponential Strategies}

Suppose a resource can offer a price from $\$ 1, \cdots \$ n$. Here, we assume the price is the net income where the cost has been deduced.

For simplicity, we first consider such a scenario: There are only two resources, $A$ and $B$, and they happen to have 


\begin{tabular}{cc|ccc} 
& & \multicolumn{3}{c}{$\mathrm{B}$} \\
$\mathrm{A}$ & & 1 & 2 & 3 \\
\hline & 1 & $(1 / 2,1 / 2)$ & $(1,0)$ & $(1,0)$ \\
& 2 & $(0,1)$ & $(1,1)$ & $(2,0)$ \\
& 3 & $(0,1)$ & $(0,2)$ & $(3 / 2,3 / 2)$
\end{tabular}

(a) Initial payoff matrix of resource game

\begin{tabular}{cc|ccc} 
& & \multicolumn{3}{c}{$\mathrm{B}$} \\
\\
& & 1 & 2 & 3 \\
\hline & 1 & $(1 / 2,1 / 2)$ & $(1,0)$ & $(1,0)$ \\
$\mathrm{A}$ & 2 & $(0,1)$ & $(1,1)$ & $(2,0)$ \\
& 3 & $(0,1)$ & $(0,2)$ & $(3 / 2,3 / 2)$
\end{tabular}

(b) Resultant matrix after $A$ removes the strictly dominated strategy 3

\begin{tabular}{cc|ccc} 
& & \multicolumn{3}{c}{ B } \\
& & 1 & 2 & 3 \\
\hline & 1 & $(1 / 2,1 / 2)$ & $(1,0)$ & $(1,0)$ \\
A & 2 & $(0,1)$ & $(1,1)$ & $(2,0)$ \\
& 3 & $(0,1)$ & $(0,2)$ & $(3 / 2, \mid 3 / 2)$
\end{tabular}

(c) Resultant matrix after $B$ removes the strictly dominated strategy 3

\section{Figure 1. Find Nash Equilibrium based on Iterated Strict Dominance}

the same reputations. Hence, the price they offered uniquely determines who will be the winner. When they offer the same price, the user may randomly select any one, so their expected profit is the half of the price. Let us assume the maximum price they can offer is $\$ 3$. We can regard the competition between these two resources as a simple game. The payoff matrix of resource $A$ and $B$ is shown in Fig. 1 (a), where each resource has three strategies each of which corresponds to a price they can offer. The first element of each entry is the expected profit of resource $A$, while the second one is that of resource $B$. Based on Game Theory, we can find the Nash Equilibrium of this game based on iterated strict dominance[6]: Resource $A$ 's profit vector for strategy 3 is $\left(0,0, \frac{3}{2}\right)$, while that for strategy 2 is $(0,1,2)$. Since each element of strategy 3 is less than that of strategy 2 , strategy 3 is strictly dominated. Hence, resource $A$ should never select strategy 3 . Fig. 1(b) shows the resultant matrix by removing strategy 3 of resource $A$. We assume resource $B$ also knows this. Now for resource $B$, strategy 3 becomes strictly dominated by strategy 2 since the corresponding profit vector $(0,0)<(0,1)$. Hence, $B$ should never choose strategy 3 , and the resultant matrix is shown in Fig. 1(c). Repeatedly, the final strategy for $A$ and $B$ would be strategy 1 . This process of finding iterated strict dominance can be applied to the case when $n$ is set to be an arbitrary value. Therefore, our conclusion is: If playing one time of this game, the best strategy of all resources would be offering the lowest price.

Now consider a more complicated scenario: There are totally $m$ resources for competition. Each time $k$ jobs are generated by the system, and each resource node can only process one job per time. If $k \geq m$, a clever resource can select a strategy by always offering the highest price. Because the users would select those resources offering the lowest price first, after all other resources have been selected, the clever resource would be the only available one. Since there are sufficient number of un-submitted jobs, this clever resource would definitely be selected no matter how high the price it offers. Thus, this strategy can maximize the resource's profit. If $k<m$, according to the discussion of the simple game, all resources have to offer the lowest price in order to win the selection. We call this strategy Binary Strategy in which the resources would offer either the lowest or the highest price depending on the system capacity (i.e., how many jobs the system can process per time unit) and the current load of the system, (i.e., the current number of jobs produced by the system per time unit).

The system is dynamic because each user will generate its jobs independently and the length of each job is not identical. Hence, it is hard for resources to accurately measure the current load of system and the number of available resources. In this dynamic system Binary Strategy may not be appropriate. Therefore, we modify the Binary Strategy and get an Exponential Strategy. In Exponential Strategy, each resource tries to measure the current load and compare it with the capacity of system. The price a resource offers is an exponential function of the difference between the current load and the system capacity. The idea is that if the current load is sufficiently larger or less than the system capacity, the resource would be more likely to choose the highest or lowest price since it is more confident about its measurement accuracy of the current load. The following equation shows how to compute the price in Exponential Strategy:

$$
p r=p r_{\text {max }} \cdot \frac{1}{1+e^{-k(x-a)},}
$$

where $p r_{\max }$ is the highest price the resources can offer, $x$ is the measured load, $a$ is the predetermined system capacity, and $k$ is a factor used to adjust the slope of the function. The greater the value of $k$, the closer the function to be binary.

\subsubsection{Historical Strategy}

Binary Strategy and Exponential Strategy are only concerned about the current load and system capacity. However, there exists other information, e.g., the previous prices offered by other competitors, which can be used by the resources to determine their price. Here, we propose an estimation-based strategy, called Historical Strategy. The idea is a resource knows the previous prices of its competitors when this resource once acted as user and had transactions with those competitors. Hence, one can estimate the possible price its competitors may offer at the next time based on their previous prices.

Assume two resources $y_{1}$ and $y_{2}$ are competing with each other. User $x$ would select one of them by comparing their appraisals $v\left(x, y_{1}\right)$ and $v\left(x, y_{2}\right)$. To win the competition with $y_{2}, y_{1}$ needs to estimate $\hat{v}\left(x, y_{1}\right)$ and $\hat{v}\left(x, y_{2}\right)$, the appraisals of user $x$ to both of them, and determine its price 
$\operatorname{pr}\left(y_{1}\right)$ to make $\hat{v}\left(x, y_{1}\right)<\hat{v}\left(x, y_{2}\right)$. In this strategy,

$$
\hat{v}\left(x, y_{1}\right)=\operatorname{pr}\left(y_{1}\right) \cdot\left(1-\hat{\operatorname{tr}}\left(x, y_{1}\right)\right),
$$

where $\hat{t r}\left(x, y_{1}\right)$ is the estimated trust of $x$ to $y_{1}$, which can be easily computed by $y_{1}$, since it knows the quality of every transaction between itself and $x$. Meanwhile, we have

$$
\hat{v}\left(x, y_{2}\right)=\hat{p r}\left(y_{2}\right) \cdot\left(1-\hat{t r}\left(x, y_{2}\right)\right)
$$

where $\hat{\operatorname{tr}}\left(x, y_{2}\right)=\operatorname{tr}\left(y_{1}, y_{2}\right)$, which means $y_{1}$ estimates $x$ 's trust to $y_{2}$ using its own one, because it assumes $y_{2}$ provides the services with similar qualities to all users. Further, $y_{1}$ estimates $y_{2}$ 's price as follows:

$$
\hat{p r}\left(y_{2}\right)=\frac{\sum_{i=1}^{n} \beta_{i} \cdot p r_{i}\left(y_{1}, y_{2}\right)}{\sum_{i=1}^{n} \beta_{i}},
$$

where $\beta$ is a decay factor, and $p r_{i}\left(y_{1}, y_{2}\right)$ denotes the $i$-th previous price of $y_{2}$ that is stored by $y_{1}$ and the first one is the most recent price.

\section{Performance Evaluation}

\subsection{Evaluation Metrics}

To evaluate the performance of our approach, we measure the following metrics:

1. User Satisfaction: It is defined as the ratio of the amount of jobs deemed successful by all users over the total amount of jobs run within every equal-length period of time. This will help us determine whether or not malicious resources have any impact on the job scheduling process.

2. Average Resource Reputation: It is defined as the average value of the reputations of all users for every resource at a given time instant. This metric is valuable to help users to differentiate various types of malicious resources.

3. Average Number of Times Resource Selected: It measures the average number of times each type of resources have ever been selected. This is another value that helps us determine if users are selecting reliable resources at a greater frequency than malicious ones.

4. Average Resource Earnings: It measures the average amount of income any resource of every type has earned. It allows us to identify whether or not incentives are being applied to the appropriate resources. In addition, it helps us to identify what the most profitable strategy is for an normal resource.

\subsection{Simulation Setup}

Our simulation consists of 100 nodes including 20 resources and 80 references. All nodes can act as user. For simplicity, we only have one type of job for which all resources are the same. Every time slot, called a tick, each user generates a number of jobs. The interval between the
Table 1. Configuration of Simulation Setup

\begin{tabular}{|c|c|}
\hline Node Types & Number \\
\hline Historical Strategy Resource / Reference & $3 / 21$ \\
\hline Exponential Strategy Resource / Reference & $3 / 21$ \\
\hline Binary Strategy Resource / Reference & $3 / 21$ \\
\hline Honest Resource / Reference & $2 / 1$ \\
\hline Naive Resource / Reference & $2 / 4$ \\
\hline Random Hypocritical Resource / Reference & $2 / 4$ \\
\hline Structured Hypocritical Resource / Reference & $2 / 4$ \\
\hline Collaborative Resource / Reference & $3 / 4$ \\
\hline
\end{tabular}

jobs generated by each user satisfies an exponential distribution, whose parameter determines whether it is a normal load or heavy load in our system. The length of each job is a random number drawn from $(0,10]$ with an average job length of 5 ticks. We assume each resource can process 3 jobs simultaneously. Hence, the whole system can process $20 \times 3$ jobs in 5 ticks, which means the system capacity is 12 jobs/tick. We set the satisfactory threshold as 0.75 . The quality of each job processing is randomly determined depending on whether the resource is reliable or malicious. For each job processed by a reliable resource, its job rating is randomly drawn from $[0.75,1]$ with an average rating 0.875 , while for a malicious resource, the job rating is always 0.01 . The lowest and highest price a resource can offer are $\$ .01$ and $\$ 100$, respectively. We set $\alpha=\beta=0.9$.

TABLE 1 lists the configuration of simulation setup in which the system experiences a normal load of 50\% resource utilization. In this configuration, we attempt to spread out the strategic resources evenly while attempting to keep the total number of potentially malicious nodes as a minority. The probability for random hypocritical nodes being malicious is $20 \%$, and the pattern for structured hypocritical nodes is to be malicious every four jobs. The purpose of the collaborative nodes is to provide malicious services to the non-group members. If the user is not within the same collaborative group as the resource and reference, the resource and reference will offer the lowest price and perform maliciously. Otherwise, they act normally and choose the historical strategy.

\subsection{Simulation Results}

Fig. 2 shows that our approach offers high user satisfactions over both resource and reference very quickly. The user satisfactions increase above $90 \%$ only after 2000 ticks, which indicates that user nodes are using reliable resources and references before they are forced to use malicious ones or wait for available reliable ones. In our simulations, we discovered that regardless of the job load in the system, user satisfactions remain constant (The results for heavy load are not provided here.).

Fig. 3 illustrates how fast the average reputation of each type of resources converges. The metric is only calculated based on the job ratings of those users having experience with resources. The figure shows our approach 
differentiates malicious resources quickly. In our simulation, the job rating for a reliable resource is 0.875 in average, while that for a malicious one is 0.01 . Hence, the average reputation of a binary, historical, exponential strategy and honest resources converges to around 0.9, while that of naive ones converges to 0.01 . For a hypocritical resource, no matter whether it is random or structured one, it is malicious with a probability $20 \%$. Hence, it must gain a rating of 0.01 with a probability $20 \%$ and a rating of 0.875 with a probability $80 \%$. Its reputation converges to $0.01 \times 20 \%+0.875 \times 80 \% \simeq 0.7$, which is consistent with the simulation results. The case of collaborative resources is a bit more complicated than the others. A collaborative resource would be malicious to non-members and reliable to members. In our simulation configuration, a collaborative resource has 93 non-member nodes who are likely to give it a rating of 0.01 and 6 member nodes giving it a rating of 0.875 . Hence, the reputation of collaborative nodes converges to $0.01 \times \frac{93}{99}+0.875 \times \frac{6}{99} \simeq 0.07$.

In Fig. 4, we show under a normal load how the average number of times resource selected increases as time goes by. Clearly, the honest resources are selected most often as they are reliable and always offer the lowest price. The same result can be expected for the binary nodes because there is no enough resource utilization to trip them offering the highest price, therefore, they effectively act as honest ones. The historical resources are selected considerably less due to the fact it offers a slightly higher price, thus, they are the next in line to be selected by users after the honest and binary ones are filled. Both types of hypocritical resources are only $80 \%$ as reliable as the historical ones, so it is reasonable to see that they are selected $20 \%$ less than the historical ones. In the long term, the collaborative resources will only be selected by its own members. Therefore, their curve is linear but only $6 \%$ as the honest and binary ones are because only 6 users are able to utilize them positively. The exponential ones can be selected after the users filter out the malicious ones. Due to their higher price, they are selected only after the historical resources are filled and the users have had enough bad experiences with the hypocritical resources.

Fig. 5 shows the average income that each type of resources has earned. Interestingly, although the exponential resources are selected the fewest amount of times of all

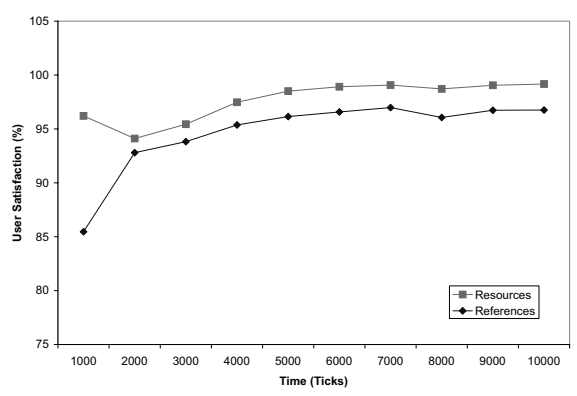

Figure 2. User Satisfaction of the reliable ones, they yield the most income of all the nodes. That means, Exponential Strategy is the best to motivate the resources to provide high-quality services in such a dynamic system. The honest resources have stabilized on a low price, although slightly higher than that of honest and binary, therefore, they receive greater income. Both hypocritical nodes use the same pricing strategy as that the historical resources do, but they are selected less often due to the $20 \%$ chance of being malicious. The collaborative resources are basically the historical resources to a much smaller audience, therefore, they are going to have even less income. The income of the honest and binary strategy nodes is equivalent to $1 / 100$ of their selection frequency because they earn $\$ 0.01$ every time selected.

\section{Summary}

In this paper, we proposed an adaptive reputation-based trust framework for peer-to-peer applications. Besides malicious nodes, we assume there exist economic nodes who compete with each other to maximize their profit. Different from traditional reputation-based trust systems, we categorized the roles of peers into three: users, references, and resources. A peer can take multiple roles at the same time within its application context. We defined four basic quantifiable metrics: job satisfactory rating, reputation, trust, and appraisal. The metric trust can be used to quantify the trustworthiness of peers and provide a trustable way of resource scheduling/selection and access control for peer-to-peer applications. We select appropriate resource to serve the user's request by quantifying and comparing the appraisal of the resources. The simulation results show that our framework supports economic resources to achieve high user satisfaction, differentiates malicious nodes from normal ones, and encourages the resources to provide high-quality services.

\section{Acknowledgment}

The research is supported in part by Carver Trust Foundation.

\section{References}

[1] A. Abdul-Rahman and S. Hailes, "Supporting Trust in Virtual Communities", in 33rd Annual Hawaii International Conference on System Sciences (HICSS-33), 2000.

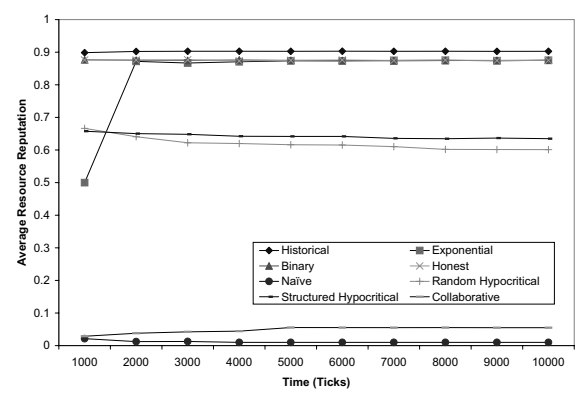

Figure 3. Average Resource Reputation 


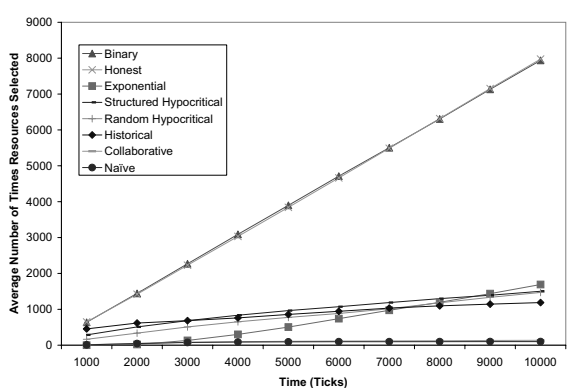

\section{Figure 4. Average Number of Times Resource Selected}

[2] K. Aberer, "P-grid: A Self-Organizing Access Structure for P2P Information Systems", in 9th International Conference of Cooperative Information Systems (CoopIS), 2001.

[3] K. Aberer and Z. Despotovic, "Managing Trust in a PeerTo-Peer Information System", in Proc. of ACM International Conference on Information and Knowledge Management (CIKM), 2001.

[4] F. Azzedin and M. Maheswaran, "Evolving and Managing Trust in Grid Computing Systems", in IEEE Canadian Conference on Electrical \& Computer Engineering (CCECE), 2002.

[5] I. Clarke, O. Sandeberg, B. Wiley, and T. W. Hong, "Freenet: A Distributed Anonymous Information Storage and Retrieval System", in Workshop on Design Issues in Anonymity and Unobservability, 2000.

[6] D. Fudenberg and J. Tirole, Game Theory, ISBN 0262061414, MIT Press, 1991.

[7] P. Golle, K. Leyton-Brown, and I. Mironov, "Incentives for Sharing in Peer-to-Peer Networks", in Proc. of Electronic Commerce, 2001.

[8] "Gnutella", Web page [Online], Available: http://www.gnutella.com.

[9] T. Johnson and P. Krishna, "Lazy Updates for Distributed Search Structure", in ACM International Conference on Management of Data (SIGMOD), 1993.

[10] S. Kamvar, M. Schlosser, and H. Garcia-Molina, "The Eigentrust Algorithm for Reputation Management in P2P Networks", in Proc. of the Twelfth International World Wide Web Conference, Budapest, 2003.

[11] "Kazaa", Web page [Online], Available: http://www.kazaa.com.

[12] S. Marsh, "Formalising Trust as a Computational Concept", in PhD Thesis, Department of Mathematics and Computer Science, University of Stirling, 1994.

[13] D. H. McKnight and N. L. Chervany, "The Meanings of Trust", in Technical Report WP9604, University of Minnesota Management Information Systems Research Center, 1996.

[14] L. Ramaswamy and L. Liu, "Free Riding: A New Challenge to Peer-to-Peer File Sharing Systems", in 36th Annual Hawaii International Conference on System Sciences (HICSS), 2003.

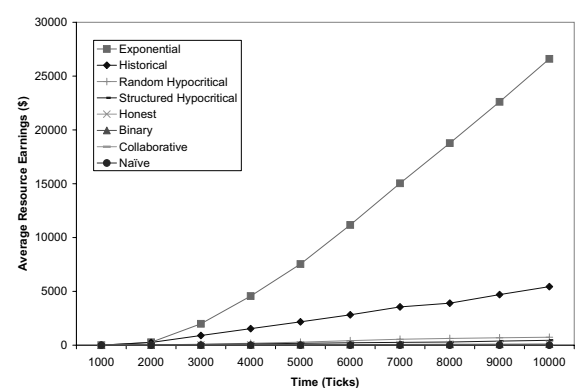

Figure 5. Average Resource Earnings

[15] S. Ratnasamy, P. Francis, M. Handley, R. Karp, and S. Shenker, "A Scalable Content Addressable Network", in ACM SIGCOMM, 2001

[16] A. Rowstron and P. Druschel, "Pastry: Scalable, Decentralized Object Location and Routing for Large-Scale PeerTo-Peer Systems", in Middleware 2001, IFIP/ACM International Conference on Distributed Systems Platforms, 2001.

[17] S. Saroiu, P. K. Gummadi, and S. D. Gribble, "A Measurement Study of Peer-to-Peer File Sharing Systems", in Proc. of Multimedia Computing and Networking (MMCN), 2002.

[18] A. Selcuk, E. Uzun, and M. Pariente, "A ReputationBased Trust Management System for P2P Networks", in 4th IEEE/ACM International Symposium on Cluster Computing and the Grid (CCGRID), 2004.

[19] S. Song and K. Hwang, "Trusted Grid Computing with Security Assurance and Resource Optimization", in Proc. of the 17th International Conference on Parallel and Distributed Computing Systems (ICPDCS), 2004.

[20] I. Stoica, R. Morris, D. Karger, F. Kaashoek, and H. Balakrishnan, "Chord: A Scalable Peer-To-Peer Lookup Service for Internet Applications”, in ACM SIGCOMM, 2001.

[21] V. Swarup and J. T. Fabrega, "Trust: Benefits, Models, and Mechanisms", in Secure Internet Programming: Security Issues for Mobile and Distributed Objects, LNCS, 1999.

[22] H. Yokota, Y. Kanemasa, and J. Miyazaki, "Fat-btree: An Update-Conscious Parallel Directory Structure", in 15th International Conference on Data Engineering, 1999.

[23] B. Yu and M. P. Singh, "A Social Mechanism of Reputation Management in Electronic Communities", in 7th International Conference on Cooperative Information Agents (CoopIS), 2000.

[24] G. Zacharia, A. Moukas, and P. Maes, "Collaborative Reputation Mechanisms in Electronic Marketplaces", in 32nd Annual Hawaii International Conference on System Sciences (HICSS), 1999. 